\title{
Novel Approaches for Food Safety Management and Communication
}

2

3

4

5

6
*Corresponding author; tel-fax: +30-210-5294693; e-mail:gjn@aua.gr
George - John E., NYCHAS*1, Efstathios Z. PANAGOU1 \& Fady MOHAREB ${ }^{2}$

aLaboratory of Microbiology and Biotechnology of Foods, Dept. of Food Science \&

Technology, Agricultural University of Athens, Iera Odos 75, Athens, 11855, Greece

bThe Bioinformatics Group, School of Water, Energy and Environment, Cranfield

University, College Road, Bedford, MK43 0AL, UK 


\section{Abstract}

20 The Current safety and quality controls in the food chain are lacking or inadequately

21 applied and fail to prevent microbial and/or chemical contamination of food products,

22 which leads to reduced confidence among consumers.

23 On the other hand to meet market demands food business operators (producers,

24 retailers, resellers) and regulators need to develop and apply structured quality and

25 safety assurance systems based on thorough risk analysis and prevention, through

26 monitoring, recording and controlling of critical parameters covering the entire

27 product's life cycle.

28 However the production, supply and processing sectors of the food chain are

29 fragmented and this lack of cohesion results in a failure to adopt new and innovative

30 technologies, products and processes.

31 The potential of using Information Technologies in tandem with data science in the food

32 chain will provide stakeholders with novel tools regarding the implementation of a more

33 efficient food safety management system. 


\section{Introduction}

At the dawn of the $21^{\text {st }}$ century, the agro-food industry is facing the following main challenges: (i) having enough to eat (Food Security) and (ii) ensure that it is safe to eat (Food Safety). These objectives should be realized not only in an environment of tremendous technological progress and evolution of consumers' life-styles, but also of economic problems, in which the food industry is called to operate under seemingly contradictory market demands.

Regarding Food Safety along the food chain, it is well known to be a shared responsibility among Food Business Operators, Authorities and Consumers [1]. Thus, Food business operators are challenged to combine requirements from different stakeholders, such as government, retailers, while the international resolutions of the Uruguay Round of the General Agreement on Tariffs and Trade (GATT) in 1995 [2], recognized public health risk as the only basis for restrictions of international trade in food, into the food industry. However, within the food chain from farm to consumer, food commodities may be exposed to multiple hazards that may cause physical, biological or chemical contamination to food and consequently increase the risk of consumption of contaminated food. These risks, e.g., pathogenic bacteria [3], mycotoxins [4] biogenic amines [5] or possible carcinogenic compounds such as caramel colours [6], have created mistrust of governments and industry by the European consumer that is threatening to become a long-term problem.

Food waste and misuse has been reported [7] to be probably the greatest problem concerning food security; indeed roughly $1 / 3$ of food produced for human consumption is lost or wasted globally and within the EU more than 100 million tonnes of food are wasted annually [European Community; Food Waste [8]. Food spoilage mainly due to 
80

microbial activity [9] is one of the most significant threats to food security. Thus minimization of food loss, as well as assurance of quality and safety [10] can be considered as the ultimate goal for the food industry.

To remedy this, the food industry and other stakeholders (e.g., competent authorities, retailers) have to provide increased vigilance with regard to food safety and quality issues. Consumers need to be and feel reassured that Food industries, as well as Food authorities, are taking extra measures to guarantee the safety of foods.

The objectives set out in the White Paper on Food Safety [1] dealt with (i) improvement of the efficiency and coherence of the EU food legislation, particularly in the area of food safety, (ii) restoring consumer confidence by the above measures and improving the quality of information available to consumers, and (iii) extending the scope of the EU food regulation by developing an EU-wide nutrition policy. To achieve these objectives in the area of food safety, a number of guiding principles have been applied, namely (1) adoption of the precautionary principle, (2) extending the scope of food safety regulation across the entire food chain from 'farm to fork' including, for example relevant controls on animal feed, (3) attribution of primary responsibility for safe food production to industry producers and suppliers within the context of the EU legislation, (4) setting out clear responsibilities for public bodies by defining standards for the food industry to meet and monitoring industry compliance, (5) establishing traceability as a major responsibility in food production and a prerequisite to both food safety and effective consumer choice.

\section{Current Food Safety Management System}

2 Nowadays a wide range of chemical and microbiological analyses has been proposed to 
evaluate the quality or safety of raw or processed materials and food products [11].

Currently, the safety of food relies heavily on regulatory inspection and sampling regimes [12]. Indeed the current Food Safety Management System, although largely based on good design of processes, products and procedures, end or finished product testing (analysed for certain hazards), is considered to be the control measure of the production process (Fig. 1). This is evident in the case of microbiological food safety where specific microbiological analyses should be followed.

These microbiological analyses can be implemented with conventional microbiology (e.g., colony counting methods) or molecular based techniques that are considered more reliable and accurate $[13,14,15,16]$. Chemical analyses are also used to monitor safety and quality of foods. These analyses either microbiological or chemical have certain disadvantages, as they are (i) time-consuming providing retrospective results, (ii) costly, (iii) few require high-tech molecular tools and thus highly trained personnel, and (iv) usually destructive to test products, limiting thus their potential to be used on-, in- or atline $[14,17]$.

Furthermore, in the case of molecular tools, results may be misleading, as these techniques are focused so far on pathogenic rather than specific groups of the microbial association, which contribute to spoilage depending on storage and packaging conditions [16]. The molecular approach is also costly, as high-tech instruments are required. In addition, due to the complexity of molecular techniques, the number of verified samples/measurements in many cases is severely limited.

It is evident that end-product analyses (testing) provide only very limited information on the safety status of a food, since the presence of a hazardous organism could give an 
indication but absence in a limited number of samples is no guarantee of safety of a

107

108

109 whole production batch. Thus, finished product testing is often too little and too late.

On the other hand, efforts have been made to replace both conventional and molecular microbiological analyses with detection of biochemical changes occurring in food that could be used to assess food spoilage or safety. This approach, however, seems inadequate because it cannot sufficiently guarantee consumer protection, since $100 \%$ inspection and sampling is technically, financially and logistically impossible.

Thus it is inevitable that new strategies should be designed and implemented focusing on the management and control of the hazards in a more proactive way by implementing an effective food safety management system and/or approaches. Indeed a modern food quality and safety assurance system should not be based on end-product analyses (Fig. 1). Instead, prevention rather than inspection, through monitoring, recording and controlling of critical parameters during the entire food's life cycle should be developed and implemented. The food life cycle should be extended beyond at and post processing phase, to include, retailer and even consumer's storage and preparation facilities.

\section{Process Analytical Technology (PAT): Implementation in food industries.}

To contribute in assurance of food safety, on and post-processing food industries and food business operators focus on the implementation of an effective Food Safety Management System (FSMS) [12], which is based on controlling, monitoring, and recording the critical parameters. On the other hand, the 'accepted' wisdom in the food industry is that processes cannot be modified as there is limited understanding of the potential impact of change and therefore re-registration would be required to 
130 demonstrate that a modified process still produces the right product from the right raw

131 materials. Additionally, post-production testing is in use today as a means to reject off-

132 specification foods or to comply with certain legislative regulations, from processes that

133 might be "out of control" (Fig. 1). Process control aims to avoid any batch-to-batch

134 changes in the raw materials, process conditions and equipment.

135 The Process Analytical Technology (PAT) concept, originated from the desire of the (bio)

136 pharmaceutical industry regulators to shift product quality control towards a science-

137 based approach, is proposed for the food industries [17] aiming at the: (i) optimization

138 of food quality, (ii) reduction of food waste through a more efficient control of the

139 processes, taking into account all processing steps and integrate sensors at the Critical

140 Control Points (CCP), (iii) reduction of the risk to consumers by controlling

141 manufacturing based on process understanding.

142 PAT can be considered as a framework for: (i) designing, analysing, and controlling

143 manufacturing through timely measurements, (ii) processing of critical quality and

144 performance attributes of raw and in-process materials and processes, (iii) process

145 measurement, information management tools, feed forward-feed backward process

146 control strategies, product \& process design and optimization strategies, and (iv)

147 reducing variation in manufacturing.

148 The PAT approach will offer a solution to a broad need identified by food industries (i.e.,

149 safety \& quality of raw and in process materials), since:

$150 \quad$ - Food business operators will be better prepared to minimize risk as a result of

151 rapid identification and control of potential hazards 
- $\quad$ Food industries that rely heavily on timely preventive control measures will also

153 benefit, since they will minimize the time needed to decide on the production and

154 distribution of particular food batches

155 - $\quad$ Food producers will increase their market shares by improving their retailers'

156 and distributors' satisfaction offering novel and easy-to-use means through ICT

157 technologies and thus reassuring customers about the quality and safety of the food

158 products they are about to buy

159

160 It needs to be stressed that the high pressure exerted from stakeholders (such as

161 consumers and regulatory authorities) to the food industry to produce safe and high

162 quality products, at low cost, minimizing additives and preservatives in a sustainable

163 manner, will force food producers to constantly develop new PAT implementations, in

164 which food safety policy will be taken into consideration. Indeed PAT envisages, a

165 scientific, risk-based, holistic and proactive approach to the food industry, with a

166 deliberate design effort from product conception through commercialization, in which

167 there will be a full understanding of how product attributes and process relate to

168 product performance. There are two steps needed for such an approach; the $1^{\text {st }}$ is the

169 "Product \& Process Design and Development", in which the upfront desired product

170 performance should be defined and the Critical Quality Attributes (CQA) should be

171 identified; the $2^{\text {nd }}$ step is a continuous risk assessment and risk control with regard to

172 the impact of (i) material attributes and process parameters on product CQAs, (ii)

173 identification and control sources of variability in material and process, and (iii)

174 monitoring (continuously) and updating process to assure consistent quality. 
176 So far the limited application of PAT in food industries [17], has narrowed down to

177 predominantly focusing on non destructive analytical instruments, spectroscopic

178 sensors based on vibrational spectroscopy, e.g. NIR, fluorescence, Raman, FT-IR, or on

179 surface chemistry, e.g. hyper and multispectral devices which are becoming increasingly

180 affordable and can be associated with advanced computational processing (SVM,

181 ensemble DLS-PCA) losing however the original holistic view $[17,18]$. This view is that

182 measurements in PAT are not just an 'analytical' measurement such as $\mathrm{pH}$, water

183 activity, metabolomics through HPLC, GC or GC/MS, spectra through spectroscopy, but

184 all those measurements can be used to infer or relate to product quality with the goal to

185 (i) understanding of the process, (ii) identification of CCP, (iii) application of knowledge

186 base to control the process.

187

188 The term "analytical" in PAT is considered to be viewed broadly to include chemical,

189 physical, microbiological, mathematical and risk analysis conducted in an integrated

190 manner, in which Information Technology (IT) will have a major role to: (1) "enhance

191 understanding and control manufacturing process" promoting in this way an ideology in

192 which "quality cannot be tested into products; it should be built-in or should be by

193 design", and (2) incorporate advanced measurements related to the above mentioned

194 tools, communication systems, i.e. integration of diverse components into ubiquitous

195 and global network; achieving reliability and security in this network.

196

197 The introduction of innovative technologies in PAT approach is one of the determining

198 factors in future growth and increased competitiveness of food industries. 
Recently, some interesting analytical approaches have been forwarded for non-

destructive rapid methods, which provide means to quantitatively monitor

characteristics of food safety and quality (Fig. 2).

202 Such methods include biosensors (enzymatic reactor systems), electronic noses (sensor

203 arrays), Fourier transform infrared (FT-IR) and Raman spectroscopy, as well as imaging

204 platforms. However, due to mass data generated for each sampling point, conventional

205 and manual approaches to interpret the output can be extremely challenging. For this

206 reason, such platforms are often used in tandem with advanced statistical methods to

207 reduce the dimensionality of the initial variables to a smaller number of factors that can

208 be used as potential biomarkers for quality and safety.

209 With the evolution of data science and machine learning approaches, novel

210 computational methods emerged to rapidly provide information related to food safety

211 and quality or categorization of foods with regard to spoilage, through the development

212 of classification or regression models using spectral or imaging data for model training

213 and validation $[18,19]$.

214 Machine learning methods are generally classified into two main groups; unsupervised

215 and supervised learning. For unsupervised learning, no prior knowledge is assumed

216 about the data; in other words, samples are clustered according to their similarity in the

217 measured profiles. This includes k-means clustering, hierarchical clustering and

218 association analysis [20]. On the other hand, in supervised learning the model is trained

219 using an input learning (training) subset, in order to unravel hidden patterns within the

220 data to predict a target variable or class. The prediction can be either a nominal value

221 (classification model), or numeric value (regression model). Algorithms belonging to 
this category include neural networks, fuzzy logic, support vector machines and decision trees $[18,20,21]$.

There is, however, a need to bridge the gap between the many emerging and rather promising devices, which could be used in the food industry in tandem with the appropriate data mining and analysis $[22,23]$. The outcome of this multidisciplinary and multi-dimensional data paradigm that integrates and crosses several scientific fields and sub-disciplines, such as process chemistry development, information technology, food science, food microbiology, molecular biology, process analytical chemistry, vibrational spectroscopy, bioinformatics, machine learning, chemical engineering, process systems and control engineering [14, 23,24,], will be for the benefit of Food Safety Management System.

\section{Enhancing Food Safety Management System (FSMS) through the Information}

\section{Technology; a new dimension}

The issue of food safety is vital in recent years and although it is constantly reviewed in the light of new scientific evidence, its implementation is not always efficient in many different parts of the food chain. For example, systematic management of food safety via HACCP, GMP, etc., entails raw material selection, as well as control of conditions during processing and distribution $[14,25]$, with the latter being the weakest link of the system. Indeed, conditions during transportation and storage at retail level are out of the manufacturer's direct control and often deviate from specifications. Temperature control is completely lacking from the store to domestic storage and until the time of preparation and consumption. Some quantitative evidence is available from studies and surveys at distribution, retail and domestic level to illustrate the magnitude of the 
problem [26]. In general, it is well established that food handling and logistics, can

substantially contribute to the risk and exposure to certain food-borne hazards [26,27].

To face the weakest link in the food chain, the implementation of parameter

quantification that allows the prediction of the behaviour of pathogenic bacteria or other hazards (mycotoxins) has been introduced in the food industry [28]. It should be stressed however that there is limitation on the accumulation of many different pieces of information, which is essential (1) to understand the rational for model development, and (2) model validation (if any) under isothermal conditions. In practice, however, temperature fluctuations may be frequent throughout food storage and distribution.

To address the issue, Information Technologies (IT), such as cloud computing and storage, big data, Internet of things, mobile web in combination with barcodes and smartphones, can be used to (i) offer the possibility to easily track the processes in the production, storage, transportation, retail, and even using phases of foods, (ii) tackle the important application of food quality (including safety) during processing [29 -33].

Indeed, Information Technology can assist food producers, retailers, authorities and even consumers to take better decisions by providing them with data and tools that enhance decision-making process, consequently allowing better management of the natural resources. To achieve this Cloud-computing platforms and the real-time monitoring and extraction of data safety and quality parameters and temperature

267 profiles throughout the production chain can be of great importance. Such cloud platforms and data repositories should be coupled with appropriate web applications in order to assist producers with their investing and planning decisions. This basic concept 
and approach was adopted in Guizhou (China) province, where on the basis of the latest

271 information technology, food production enterprises, government, testing organizations

272 and consumers were integrated into a unified food safety information service cloud

273 platform [34]. The core technology of the cloud platform is composed of food safety

274 knowledge system, testing management system, food safety information publicity

275 system, as well as mobile application. The food factory inspection data, government

276 inspection data, testing organizations data and consumers purchasing information are

277 integrated into food safety and nutrient test big data. Utilizing the data to explore the

278 information that is needed by all the parties, can be served as a solution to the risk

279 exchange problem faced by food stakeholders, while at the same time, the food safety

280 problem can be solved through the contribution of different stakeholders.

282 Cloud computing can also be of great importance for the FSMS concept, as a means to

283 store information associated with each product and make this information accessible to

284 retailers and consumers via, e.g. platforms, barcodes such as QR codes (Fig. 3). Currently

285 QR codes are frequently integrated within the food packaging system to direct consumer

286 to a product web-page with more information about the given product such as origin,

287 cooking instructions and suggested recipes. However, there is great potential to expand

288 the usage of barcoding beyond simply pointing to a static web page; though tracing back

289 the product to the collection of enormous data derived from the "connected" rapid and

290 non-invasive analytical platforms within the PAT framework (Fig. 2). In this way, the

291 combination of rapid methods with machine learning and barcodes will provide a

292 valuable "real life" application of the technology in a new domain (food freshness and

293 safety) that will contribute to the predicted increase in the cloud computing market. The 
concept of IT efficiency also embraces the ideas encapsulated in green computing, since not only are the computing resources used more efficiently, but further, the computers can be physically located in geographical areas that have access to cheap electricity while their computing power can be accessed long distances away over the Internet.

Barcodes and more specifically QR codes are becoming a standard consumeradvertising tool. They have been gaining an increased popularity over the past five years with the introduction of smartphones with embedded cameras and image processing packages. Nowadays, over half of EU citizens have a smartphone capable of capturing QR codes, and around $25 \%$ of smartphone users are already familiar with the process of

304 scanning a QR code. We believe that in near future there will be a unique connection 305 between dynamic cloud-based information and QR codes that will provide enormous 306 information to food stakeholders (Fig. 3) as well as a massive boost e.g. the newer 307 technologies of mobile visual search (MVS) and near field communication (NFC) but 308 neither of these are suitable for providing additional information about specific product 309 items.

311 On the other hand the Internet-of-Things (IoT) and emerging technologies (i.e., 312 Wireless sensor network, cloud technology and machine learning) is a vision of 313 connectivity for anything, anytime and anywhere, which may have a dramatic impact on 314 our daily life as what the Internet has done in the past two decades [29]. This will have 315 significant economic impact on each of the individual information technologies as it creates a previously untapped market for these technologies, but it will also 
317 demonstrate exciting new synergies between the technologies that will spark new ideas 318 for future innovations.

319 Internet technologies allow supply chains to use virtualizations dynamically in

320 operational management processes. This will improve support for food companies

321 dealing with perishable products, unpredictable supply variations and stringent food

322 safety and sustainability requirements. Virtualization enables supply chain actors to

323 monitor, control, plan and optimize business processes remotely and in real-time

324 through the Internet, based on virtual objects instead of observations on-site.

325

326 


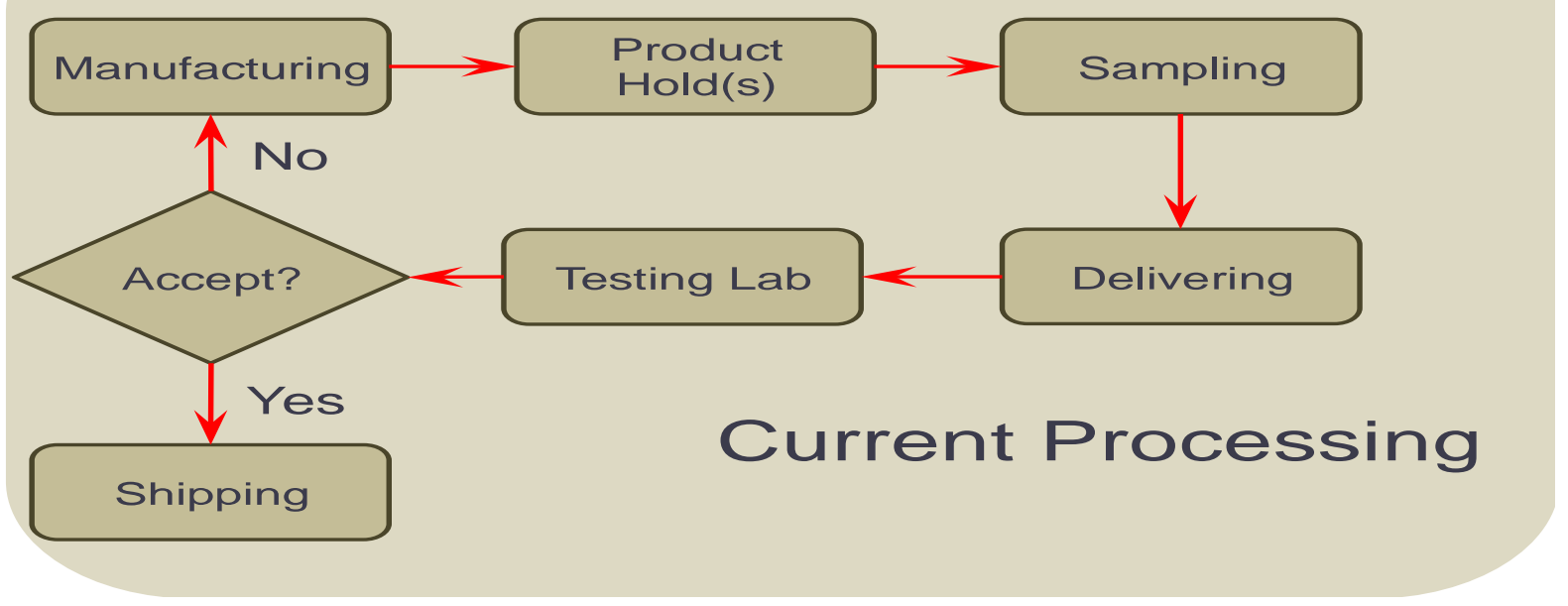

Fig. 1 Current testing and controlling of food safety

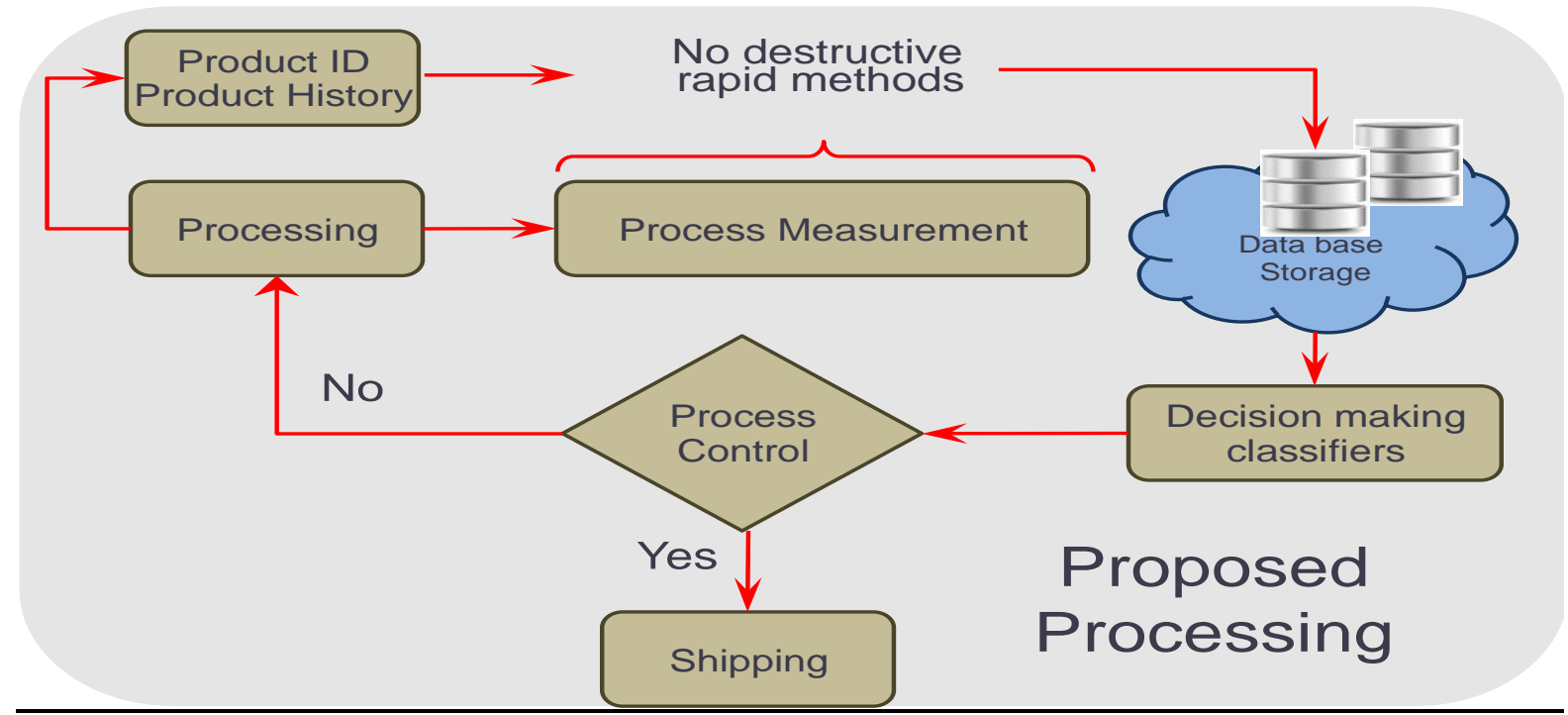

Fig 2. Use of non-destructive rapid methods for the implementation of PAT in food processing; 


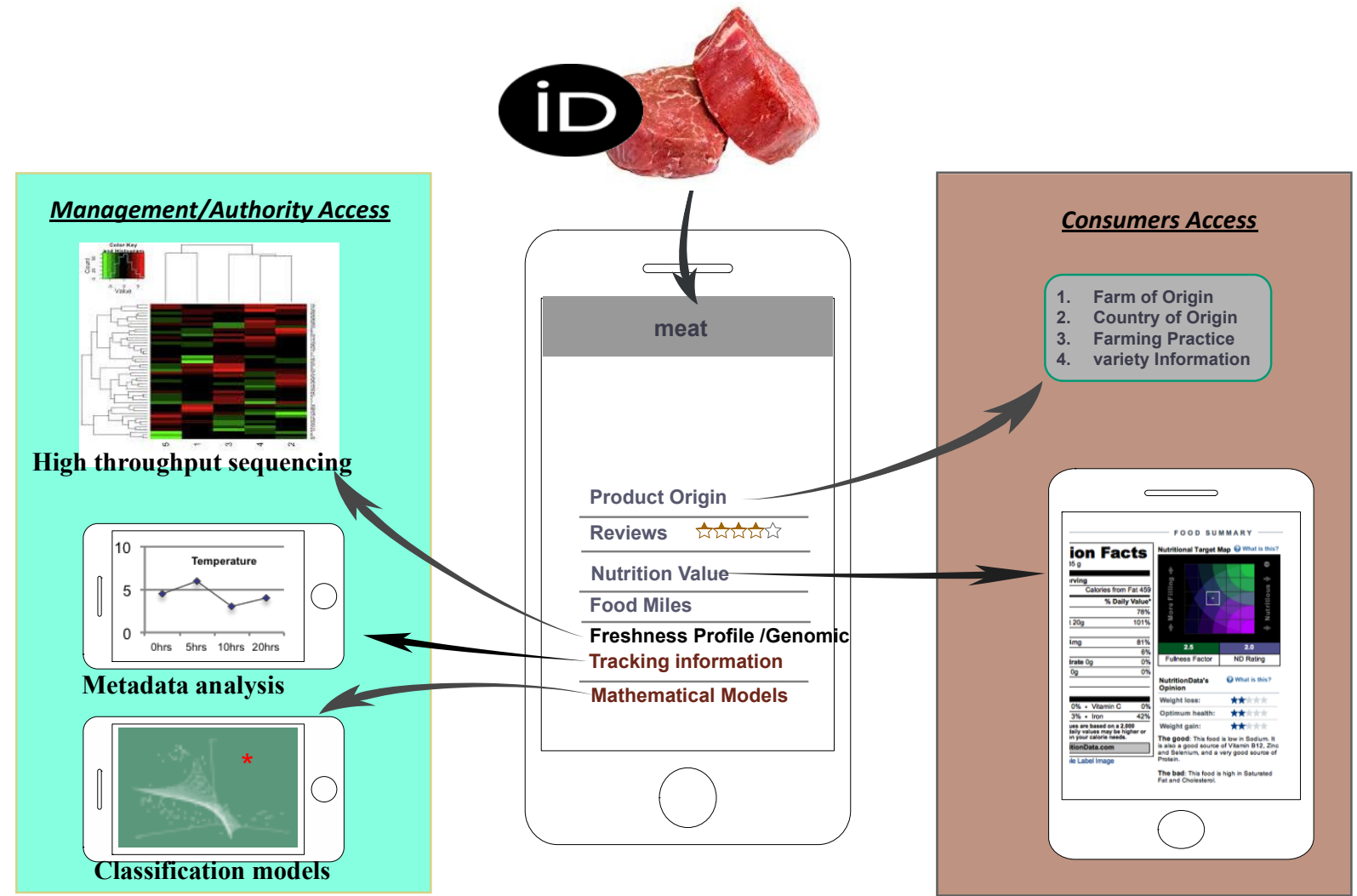


1. White Paper on Food Safety; Chapter 2 (2000) COM (1999) 719 final

2. http://www.fao.org/docrep/004/w7814e/w7814e04.htm],

3. EFSA: The European Union summary report on trends and sources of zoonoses, zoonotic agents and food-borne outbreaks in 2013 EFSA Journal 2015; 13:3991 [165 pp.]),

4- http://www.efsa.europa.eu/en/topics/topic/mycotoxins.htm] [accessed 21.03.2015]

5. EFSA - Scientific Opinion on risk based control of biogenic amine formation in fermented foods: http://www.efsa.europa.eu/de/efsajournal/pub/2393.htm EFSA Journal 2011;9(10):2393 [93 pp.]],

6. The EFSA Homepage - Caramel colours: http://www.efsa.europa.eu/en/press/news/121219.htm ]

7. FAO 2011 Global food losses and food waste; http://www.fao.org/docrep/014/mb060e/mb060e00.pdf) [accessed 19.032015]

8. http://ec.europa.eu/food/safety/food_waste/index_en.htm; [accessed 16.03.2015]

9. Gram L, \& Dalgaard P, Fish spoilage bacteria - problems and solutions. Current Opinion Biotechnology, 2002 13: 262-266.

10- EFSA Journal 2014; 12 (2):3547

11. European Commission, 2005. Commission Regulation (EC) No. 2073/2005 of Nov 2005 on microbiological criteria for foodstuffs. Off. J. Eur. Union L 338, 126.

12. Zwietering $M H$, Jacxsens $L$, Membre J-M, Nauta $M$, Peterz $M$ : Relevance of microbial finished product testing in food safety management Food Control 2016 60: 31-43

13. Velusamy V, Arshak K, Korostynska O, Oliwa K, Adley C: An overview of foodborne pathogen detection: in the perspective of biosensors. Biotechn Advances, 2010 28, 232-254. http://dx.doi.org/10.1016/j.biotechadv.2009.12.004.

14. Nychas G-JE, Skandamis PN, Tassou CC, Koutsoumanis KP: Meat spoilage during distribution. Meat Sci. 2008, 78:77-89.

http://dx.doi.org/10.1016/j.meatsci.2007.06.020.8;

15. Papadopoulou O, Panagou EZ, Tassou CC, Nychas G-J E: Contribution of Fourier transform infrared (FTIR) spectroscopy data on the quantitative determination of minced pork meat spoilage. Food Res. Int., 201144 (10), 3264e3271. http://dx.doi.org/10.1016/j.foodres.2011.09.012.1). 
16. Doulgeraki AI, Ercolini D, Villani F, Nychas G-JE: Spoilage microbiota associated to the storage of raw meat in different conditions. Int. J. Food Microbiol., 2012 157(2), 130e141. http://dx.doi.org/10.1016/j.ijfoodmicro.2012.05.020.

17. van den Berg F, Lyndgaard CB, Sørensen KM, Engelsen SB: Process Analytical Technology in the food industry Trends in Food Science Techn 2012

18. A.I. Ropodi AI, Panagou EZ, Nychas G-JE: Data mining derived from food analyses using non-invasive/nondestructive analytical techniques; determination of food authenticity, quality \& safety in tandem with computer science disciplines Trends in Food Science Techn 2016 50:11-25

19. Goodacre R, Vaidynathan S, Dunn WB, Harrigan GG, Kell, DB: Metabolomics by numbers: acquiring and understanding global metabolite data. Trends. Biotechnol. 2004 22: 245-252.

20. Harrington P: Machine learning in action. Manning Publications Co., Shelter Island, NY (2012)

21. Ellis DI, Goodacre R: Rapid and quantitative detection of the microbial spoilage of muscle foods: current status and future trends. Trends Food Sci Techn 2001 (11), 414e424. http://dx.doi.org/10.1016/S0924-2244(02) 00019-5.

22. Goodacre R: Explanatory analysis of spectroscopic data using machine learning of simple, interpretable rules. Vibrat. Spectr., 2003, 32: $33 \mathrm{e} 45$. http://dx.doi.org/10.1016/S0924-2031(03)00045-

23. Reich, G: Near-infrared spectroscopy and imaging: Basic principles and pharmaceutical applications. Advanced Drug Delivery Reviews, 2005. 57:11091143.

24. Roy, S:Quality by design: A holistic concept of building quality in pharmaceuticals. Int J Pharm Biomed Res 2012, 3: 100-108)

25. Koutsoumanis K., Pavlis A, Nychas G-JE, Xanthiakos K:Probabilistic modeling as a means for evaluating the effect of distribution, retail and domestic storage conditions on the growth of Listeria monocytogenes in pasteurized milk Appl. Environ. Microbiol. 2010 76: 2181-2191

26. Catellani P, RM Scapin RM, Alberghini L, Radu IL:Levels of microbial contamination of domestic refrigerators in Italy. Food Control, 2014 42: 257;

27. Neal JA, Binkley M, Henroid D: Assessing Factors Contributing to Food Safety Culture in Retail Food Establishments Food Prot Trends 2012 32: 468-476.

28. Tenenhaus-Aziza F, Ellouze M:Software for predictive microbiology and risk assessment: A description and comparison of tools presented at the ICPMF8 Software Fair (2015) Food Microbiol. 45, 290-299). 
29. Pang, Z., Chen, Q., Han,W., Zheng, L: Value-centric design of the internet-ofthings solution for food supply chain: Value creation, sensor portfolio and information fusion. Inf Syst Front 2015 17:289-319

30. Han W, Gu Y, Wang W, Zhang Y, Yin Y, Wang J, Zheng L-R: The design of an electronic pedigree system for food safety. Inf Syst Front 2015 17:275-287,

31. Verdouw CN, Wolfert J, Beulens AJM, Rialland A: Virtualization of food supply chains with the internet of things J. Food Engin. $2016176128 \mathrm{e} 136$,

32. Verdouw CN, Robbemonda RM, Verwaarta T, Wolferta J, Beulens AJM:A reference architecture for IoT-based logistic information systems in agri-food supply chains Enterprise Information systems, 2015 http://dx.doi.org/10.1080/17517575.2015.1072643,

33. Ying F, Fengquan L:"Application of Internet of Things to the Monitoring System for Food Quality Safety," Digital Manufacturing and Automation (ICDMA), 2013 Fourth International Conference on, Qingdao, 2013, pp. 296-298.

34. Chen K., Tan H, Gao J, Lu Y: Big data based design of food safety cloud platform (Conference Paper) Appl. Mechanics Materials 2014, 536-537, 583-587 2nd International Conference on Mechatronics, Robotics and Automation, ICMRA (http://www.scientific.net/AMM.536-537.583) 